\title{
Advanced holographic methods in extreme ultraviolet interference lithography
}

\author{
Bernd Terhalle $^{a}$, Andreas Langner ${ }^{a}$, Birgit Päivänranta ${ }^{a}$, \\ and Yasin Ekinci ${ }^{a, b}$ \\ ${ }^{a}$ Laboratory for Micro- and Nanotechnology, Paul Scherrer Institute, \\ CH-5232 Villigen PSI, Switzerland; \\ ${ }^{b}$ Laboratory of Metal Physics and Technology, Department of Materials, ETH Zürich, \\ CH-8093 Zürich, Switzerland
}

\begin{abstract}
We study the formation of complex pattern geometries and beam shapes in diffraction-based extreme ultraviolet interference lithography. In particular, we demonstrate numerically as well as experimentally the potential of interfering multiple beams with well-controlled relative phase relations for the fabrication of high resolution periodic and quasiperiodic nanostructures.
\end{abstract}

Keywords: EUV interference lithography

\section{INTRODUCTION}

Extreme ultaviolet (EUV) lithography is currently considered to be the next-generation technique for highvolume semiconductor chip production with feature sizes of $22 \mathrm{~nm}$ and below. ${ }^{1,2}$ In addition to the development of industrial projection systems, EUV interference lithography (EUV-IL) has recently been attracting growing interest as a powerful tool for academic as well as in industrial research. ${ }^{3}$ Providing various fundamental features, such as high resolution, high throughput due to a parallel fabrication process, large area or a large depth of focus, EUV-IL has been employed, e.g., for development of high-resolution resists ${ }^{4}$ or large area nanopatterning. ${ }^{5}$

In the most common experimental EUV-IL scheme, a wavefront-division interferometer consisting of two- or more transmission diffraction gratings is illuminated with a spatially coherent EUV beam. The gratings diffract the beam into multiple diffraction orders which overlap at a certain distance from the mask where they form an interference pattern. For the $1^{\text {st }}$ diffraction order it is half the period of the gratings on the mask..$^{5,6}$ In most realizations, such $1^{\text {st }}$ order diffraction is used, but also interference patterns produced with $2^{\text {nd }}$ order diffraction have been reported. ${ }^{7}$

In addition to two-beam interference for high resolution one-dimensional line/space patters the implementation of multiple-beam interference for the generation of two-dimensional patterns has been discussed for EUV-IL. ${ }^{3}$ It has been demonstrated that the observed intensity pattern strongly depends on the relative phase between the interfering waves. For the above mentioned diffraction-based EUV-IL scheme, this implies precise positioning of the individual gratings in order to obtain the desired phase relations and consequently puts strong requirements on the fabrication process. However, it has been shown that such precise mask fabrication is feasible with electron beam lithography ${ }^{8}$ and thus it opens up new possibilities for the generation of complex pattern geometries by phase-engineered EUV-IL.

In the visible wavelength region, the structural variety of multiple-beam interference patterns with controlled phase relations between the interfering waves has recently been discussed in the context of discrete nondiffracting wave fields. ${ }^{9}$ Here, we adapt this concept to diffraction-based EUV-IL and demonstrate its potential as a general approach for the generation of complex, high resolution periodic as well as quasiperiodic nanostructures. Sec. 2 describes the basic concepts of phase-engineered EUV-IL. These concepts are subsequently applied in numerical simulations as well as experiments for the generation of periodic structures by four- and six-beam interference in Sec. 2.1. Finally, we extend the analysis to more complex structures by realizing quasiperiodic five- and eight-beam interference patterns in Sec. 2.2.

Further author information: Send correspondence to Yasin Ekinci; E-mail: yasin.ekinci@psi.ch

Nanoengineering: Fabrication, Properties, Optics, and Devices VIII, edited by Elizabeth A. Dobisz, Louay A. Eldada, Proc. of SPIE Vol. 8102, 81020V · C 2011 SPIE · CCC code: 0277-786X/11/\$18 · doi: 10.1117/12.893733 


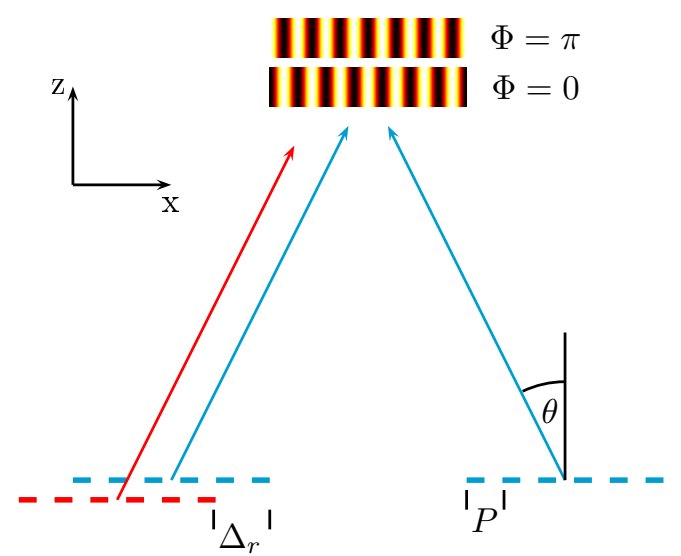

Figure 1. Schematic illustration of phase-engineered two-beam interference. Shifting one grating by $(n+1) P / 2$ induces a relative phase shift of $\Phi \propto \pi$ between the interfering waves and thus a transverse shift in the resulting interference pattern.

\section{PHASE-ENGINEERED MULTIPLE-BEAM EUV INTERFERENCE LITHOGRAPHY}

We consider $N$ interfering plane waves having their individual wave vectors $\mathbf{k}_{n}$ located in Fourier space on a cone with an opening angle $2 \theta$ which is defined by the transverse wave vector component $k_{t}=\sqrt{k_{x}^{2}+k_{y}^{2}}$ as

$$
k_{t, n}=\left|\mathbf{k}_{n}\right| \sin (\theta) .
$$

Adding up the amplitudes of the interfering waves, the resulting field distribution can be written as:

$$
U_{t o t}=\sum_{n}^{N} U_{0, n} \exp \left(i\left(k_{x, n} x+k_{y, n} y+k_{l, n} z\right)\right)
$$

where $k_{l, n}=\sqrt{|\mathbf{k}|^{2}-k_{t, n}^{2}}$ defines the longitudinal wave vector component.

As already mentioned in the introduction, such multiple beam interference is commonly realized in EUVIL by using a mask containing several transmission diffraction gratings with period $P$ and overlapping the corresponding first diffraction orders in the substrate plane. ${ }^{5}$ In order to satisfy the above mentioned conditions, the centers of the gratings should be arranged on a circle whose radius is determined by the grating period $P$ and the gap between mask and substrate. Consequently, the opening angle of the Fourier space cone formed by the $\mathbf{k}$ vectors of the interfering waves is determined by the diffraction angle:

$$
\theta=\arcsin (\lambda / P) .
$$

As illustrated in Fig. 1 for the simple case of $N=2$, phase control in this scheme can be achieved by introducing a radial shift $\Delta_{r, n}$ of one or more gratings, i. e. a shift in the direction perpendicular to the grating lines. The path difference resulting from the shift of a single grating can be written as

$$
\phi_{n}=\Delta_{r, n} \sin (\theta)=\Delta_{r, n} \lambda / P .
$$

As expected, a shift of one period $P$ therefore results in a relative phase difference of $2 \pi$. Including such radial shifts, the modified field distribution reads as

$$
U_{t o t}=\sum_{n}^{N} U_{0, n} \exp \left(i\left(k_{x, n}\left(x-\Delta_{x, n}\right)+k_{y, n}\left(y-\Delta_{y, n}\right)+k_{l, n} z\right)\right),
$$



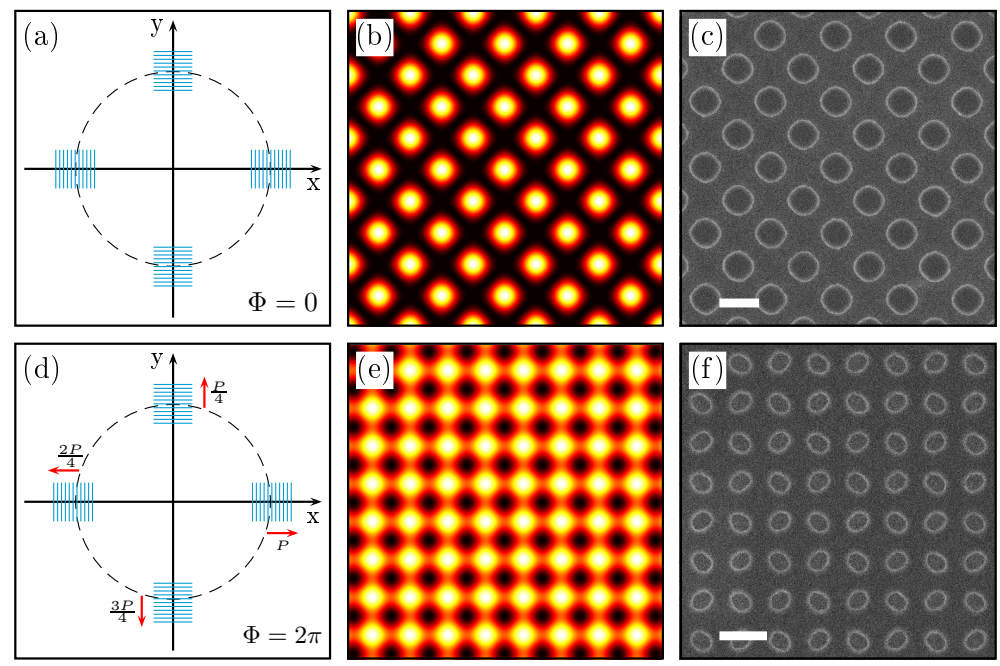

Figure 2. Four-beam interference for the case of $\Phi=0$ (top) and $\Phi=2 \pi$ (bottom). (a), (d): Sketch of the mask design. Red arrows in (d) indicate the radial shift of the individual gratings with respect to the original positions in (a); (b),(e): Simulated intensity distributions; (c), (f): Scanning electron micrographs of the experimentally recorded patterns in HSQ resist. The scale bars correspond to $200 \mathrm{~nm}$.

with $\Delta_{x, n}=\Delta_{r, n} k_{x, n} / k_{t, n}$ and $\Delta_{y, n}=\Delta_{r, n} k_{y, n} / k_{t, n}$. Although Eq. 5 in principle allows for the generation of an infinite number of patterns produced by arbitrary arrangements of gratings centered on a circle on the mask, we will restrict our following analysis to $N=4,5,6$, and 8 with gratings being centered on the vertices of $N$-fold equilateral polygons.

\subsection{PERIODIC PATTERNS}

Two-dimensional periodic intensity distributions can be obtained by interfering either two, three, four or six beams. However, as can easily be shown by analysing Eq.(5), two- and three-beam interference patterns are independent of the relative phases of the interfering waves, except for a transverse shift of the interference pattern. $^{8,9}$

Therefore, we start by considering the case of four-beam interference as illustrated in Fig. 2. Without any additional phase shift [Fig. 2(a)], the four beams interfere constructively at points on a $45^{\circ}$ rotated square grid as shown by the simulated intensity distribution in Fig. 2(b). In contrast, introducing a relative phase of $\Phi=2 \pi$, e. g. by shifting the gratings by $n P / 4$ with $n=1,2,3,4$ as illustrated in Fig. 2(d), the interference pattern shows a different intensity distribution which is equivalent to the incoherent addition of two crossed gratings [Fig. 2(e)]. It should be noted that the arrangement of gratings used in Fig. 2(d) is not the only solution to generate this pattern. The presented general approach with an accumulated total phase shift of $2 \pi$ implies a relative phase difference of $\pi$ between opposite gratings such that the pattern shown in Fig. 2(e) could for example also be achieved by simply shifting one single grating by half a period [cf. Fig. 1].

In order to realize the discussed patterns in experiment, we use a mask containing two sets of gratings according to the schematic illustrations in Figs. 2(a) and (c). The mask is fabricated on a $100 \mathrm{~nm} \mathrm{Si}_{3} \mathrm{~N}_{4}$ membrane with a thermally evaporated $\mathrm{Cr}$ film of $40-70 \mathrm{~nm}$ thickness which was spin coated with a $70 \mathrm{~nm}$ thick layer of polymethylmethacrylate (PMMA). The gratings were written by electron beam lithography with nanometer scale accuracy of the position for each grating. The developed patterns were finally transferred into the Cr layer by a dry etching process. The corresponding exposure was performed at the XIL-II beamline at the Swiss Light Source (SLS) which provides a spatially coherent beam at a wavelength of $13.5 \mathrm{~nm}$. Figs. 2(c) and (f) show scanning electron micrographs of patterns for both cases obtained in negative tone hydrogen silsesquioxane (HSQ) resist.

In a next step, we study the influence of relative phase shifts on the case of six-beam interference as shown numerically in Fig. 3. For $\Phi=0$, i. e. all interfering waves having equal phase [Figs. 3(a),(d)], the well known 

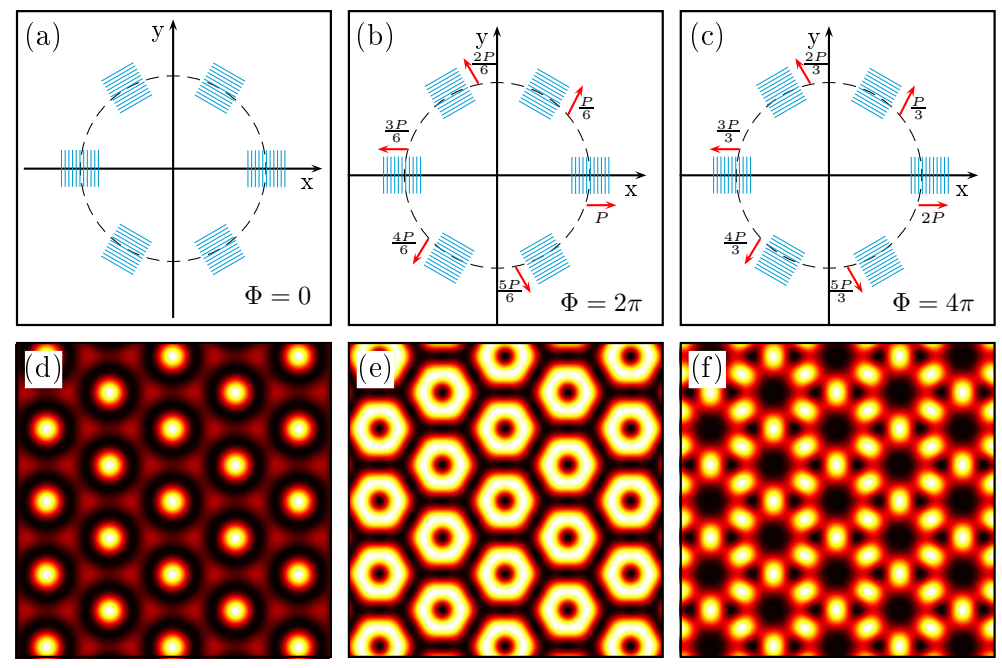

Figure 3. Numerical simulation of six-beam interference for the case of $\Phi=0$ [(a) and (d)], $\Phi=2 \pi[(\mathrm{b})$ and (e)], and $\Phi=4 \pi[(\mathrm{c})$ and (f)]. (a)-(c): Sketch of the mask design. Red arrows indicate the radial shift of the individual gratings with respect to the original positions in (a); (d)-(e)): Simulated intensity distributions.
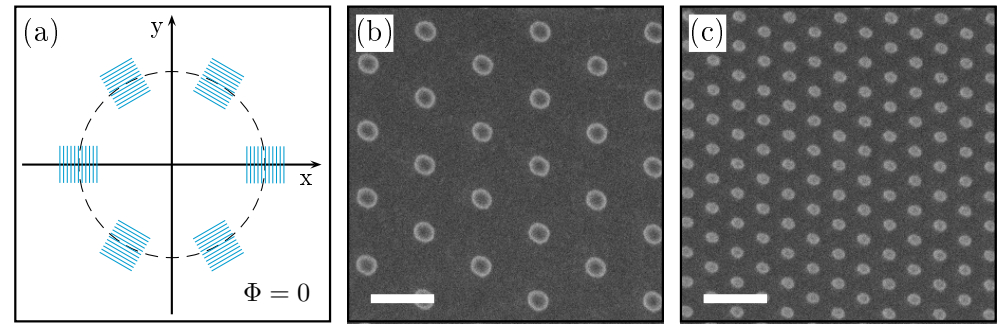

Figure 4. Experimental realization of six-beam interference for $\Phi=0$. (a): Sketch of the mask design; (b), (c): scanning electron micrographs of the experimentally recorded patterns in HSQ resist for $P=200 \mathrm{~nm}$ in (b) and $P=88 \mathrm{~nm}$ in (c). The scale bars correspond to $200 \mathrm{~nm}$.

hexagonal structure is obtained. Inducing a relative phase of $\Phi=2 \pi$ changes the pattern to a hexagonal arrangement of honeycomb-like intensity shapes as illustrated in Figs. 3(b) and (d). The change of the pattern becomes even more drastic for $\Phi=4 \pi$, in which case the interference results in the so-called kagome structure ${ }^{10,11}$ depicted in Fig. 3(f).

For the experimental generation of six-beam interference patterns in our EUV-IL setup, we again fabricated the corresponding mask on a $100 \mathrm{~nm}$ thick $\mathrm{Si}_{3} \mathrm{~N}_{4}$ membrane with a thermally evaporated $\mathrm{Cr}$ film. This time, the membrane was spin coated with an HSQ layer of approximately $20 \mathrm{~nm}$ thickness, exposed by electron beam lithography and the pattern again transferred into the Cr layer by a dry etching process. Fig. 4 exemplarily shows the obtained hexagonal patterns $(\Phi=0)$ for two different periods. Simlar to the four-beam interference, we again observe very good agreement between numerical simulations and experiment.

\subsection{QUASIPERIODIC PATTERNS}

In contrast to the previously discussed cases of two-, three-, four-, and six-beam interference, the obtained patterns for $N=5,7,8, \ldots$ are no longer periodic but can be characterized as quasiperiodic. Such quasiperiodic structures are unique in showing a long-range aperiodic order and rotational symmetries outside the traditional crystallographic classification scheme. ${ }^{12}$ Following their first discovery in alloy systems, ${ }^{13}$ quasiperiodic structures have been studied in different fields of physics due to their fascinating properties. In particular, artificial quasiperiodic photonic crystals attracted great interest after it had been demonstrated that they possess more isotropic band gaps than their periodic counterparts and thus enable a variety of interesting wave propagation 

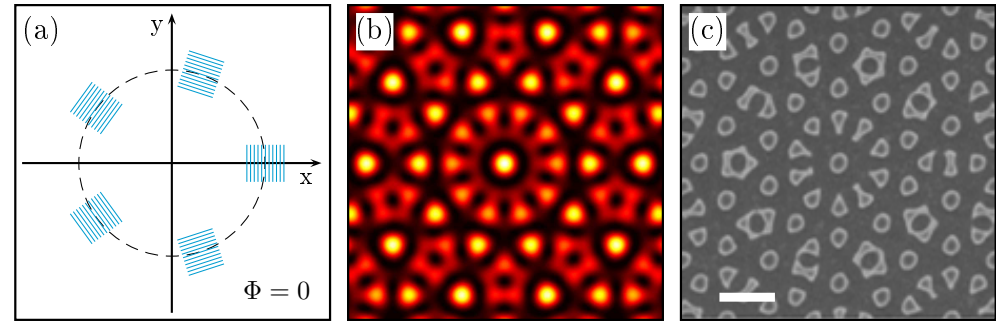

Figure 5. Generation of quasiperiodic penrose nanostructures by five-beam interference $(\Phi=0)$. (a): Sketch of the mask design. (b): Simulated intensity distribution; (c): Scanning electron micrograph of an experimentally recorded pattern in HSQ resist. The scale bar corresponds to $200 \mathrm{~nm}$.
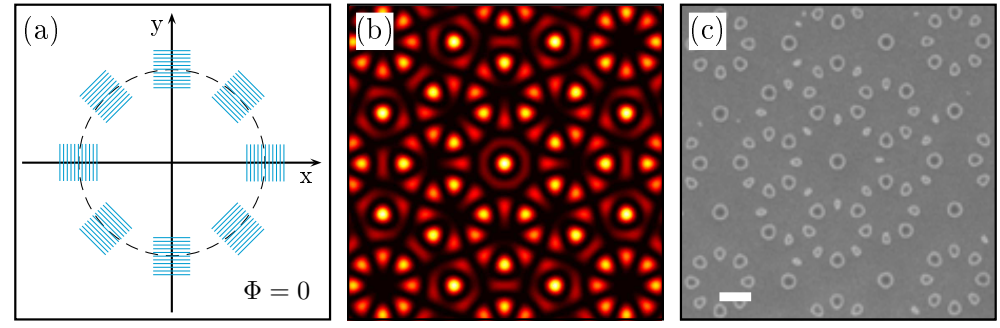

Figure 6. Generation of quasiperiodic nanostructures by eight-beam interference $(\Phi=0)$. (a): Sketch of the mask design. (b): Simulated intensity distribution; (c): Scanning electron micrograph of an experimentally recorded pattern in HSQ resist. The scale bar corresponds to $200 \mathrm{~nm}$.

phenomena. ${ }^{14,15}$ The fabrication of quasiperiodic nanostructures is difficult to achieve in serial writing processes due to their nonperiodic translational order. Time consuming calculations and a huge amount of data are necessary to mimic the desired structure as close as possible. A much easier and inherently accurate approach is given by use of the presented multiple-beam interference scheme as we have recently demonstrated. ${ }^{16}$ Below, we will exemplarily demonstrate results for five- and eight-beam interference.

Fig. 5 summarizes our results for the case of five-beam interference leading to a representation of the wellknown Penrose pattern [Fig. 5(b)]. Interestingly, additional phase shifts in this scheme only cause a rearrangement of the basic five-fold building blocks and thus hardly influence the totally observed intensity distribution. ${ }^{9}$ Similar to the six-beam interference case, we fabricated a grating mask with a design as illustrated in Fig. 5(a). Comparing the results shown in Figs. 5(b) and (c) reveals that the experimental result obtained in HSQ resist [Fig. 5(c)] nicely reproduces the simulated intensity distribution of the penrose pattern [Fig. 5(b)].

In addition, the same fabrication process was employed for an eight-beam interference mask for the realization of quasiperiodic eight-beam interference patterns as demonstrated in Fig. 6. Again, we observe a very good agreement between numerical simulations and experimentally obtained pattern, both clearly revealing the eightfold symmetry of the desired pattern.

We would like to point out the fact that increasing the number of interfering waves would eventually transfom the resulting interference pattern into a Bessel beam of zeroth order for $N \rightarrow \infty .{ }^{9}$ This implies that the presented EUV-IL setup could be used for the generation of Bessel beams by using a mask containing a ring-shaped circular grating as illustrated in Fig. 7.

\section{CONCLUSIONS}

In conclusion, we have demonstrated experimentally as well as numerically the potential of phase-controlled multiple beam interference schemes in diffraction-based EUV-IL setups for the generation of high-resolution periodic and quasiperiodic nanostructures. In contrast to the simple cases of two- and three-beam interference where the resulting intensity distribution is essentially independent of the relative phase relations between the 

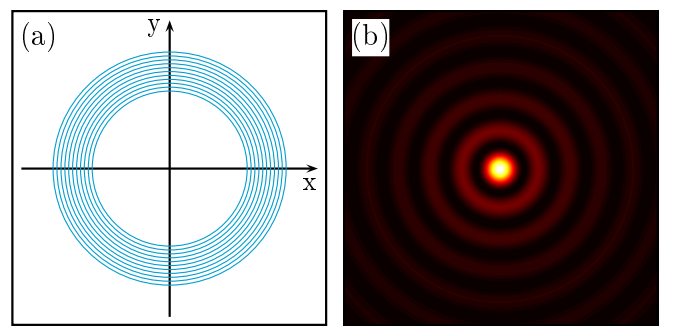

Figure 7. Generation of EUV Bessel beams. (a): Sketch of the mask design. (b): Simulated intensity distribution.

interfering beams, four- and six-beam configurations can generate a variety of interesting periodic pattern geometries. Moreover, we have shown the implementation of five- and eight-beam interference for the generation of quasiperiodic nanostructures. In particular, the five-beam configuration enables the generation of high resolution penrose structures which are expected to find applications in photonic or plasmonic applications.

\section{ACKNOWLEDGMENTS}

The authors thank Vitaliy A. Guzenko and Michaela Vockenhuber for useful discussions and technical support. Part of this work was performed at the Swiss Light Source (SLS), Paul Scherrer Institute, 5232 Villigen PSI, Switzerland.

\section{REFERENCES}

[1] Tallents, G., Wagenaars, E., and Pert, G., "Optical lithography: Lithography at EUV wavelengths," Nature Photonics 4, 809 (2010).

[2] Wagner, C. and Harned, N., "EUV lithography: Lithography gets extreme," Nature Photonics 4, 24 (2010).

[3] Solak, H. H., "Nanolithography with coherent extreme ultraviolet light," J. Phys. D: Appl. Phys. 39, R171 (2006).

[4] Langner, A., Solak, H. H., Gronheid, R., van Setten, E., Auzelyte, V., Ekinci, Y., van Ingen Schenau, K., and Feenstra, K., "Measuring resist-induced contrast loss using EUV interference lithography," Proc. SPIE 7636, 76362X (2010).

[5] Auzelyte, V., Dais, C., Farquet, P., Grützmacher, D., Heyderman, L. H., Luo, F., Olliges, S., Padeste, C., Sahoo, P. K., Thomson, T., Turchanin, A., David, C., and Solak, H. H., "Extreme ultraviolet interference lithography at the paul scherrer institut," J. Micro/Nanolith. MEMS MOEMS 8, 021204 (2009).

[6] Wei, M., Attwood, D., and Gustafson, T. K., "Patterning a 50-nm period grating using soft x-ray spatial frequency multiplication," J. Vac. Sci. Technol. B 12, 3648 (1994).

[7] B. Päivänranta, Langner, A., Kirk, E., David, C., and Ekinci, Y., "Sub-10nm patterning using EUV interference lithography," Nanotechnology 22, 375302 (2011).

[8] Solak, H. H., David, C., Gobrecht, J., Wang, L., and Cerrina, F., "Multiple-beam interference lithography with electron beam written gratings," J. Vac. Sci. Technol. B 20, 2844 (2002).

[9] Boguslawski, M., Rose, P., and Denz, C., "Increasing the structural variety of discrete nondiffracting wave fields," Phys. Rev. A 84, 013832 (2011).

[10] Boguslawski, M., Rose, P., and Denz, C., "Nondiffracting kagome lattice," Appl. Phys.Lett 98, 061111 (2011).

[11] Law, K. J., Saxena, A., Kevrekidis, P. G., and Bishop, A. R., "Localized structures in kagome lattices," Phys. Rev. A 79, 053818 (2009).

[12] Wang, X., Ng, C., Tam, W., Chan, C., and Sheng, P., "Large-area two-dimensional mesoscale quasicrystals," Adv. Mater. 15, 1526 (2003).

[13] Shechtman, D., Blech, I., Gratias, D., and Cahn, J. W., "Metallic phase with long-range orientational order and no translational symmetry," Phys. Rev. Lett. 53, 1951-1953 (1984). 
[14] Zoorob, M. E., Charlton, M. D. B., Parker, G. J., Baumberg, J. J., and Netti, M., "Complete photonic bandgaps in 12-fold symmetric quasicrystals," Nature 404, 740 (2000).

[15] Man, W., Megens, M., Steinhardt, P. J., and Chaikin, P. M., "Experimental measurement of the photonic properties of icosahedral quasicrystals," Nature 436, 993 (2005).

[16] Langner, A., B. Päivänranta, Terhalle, B., and Ekinci, Y., "Fabrication of quasiperiodic structures with EUV interference lithography," submitted (2011). 